\title{
Method for measuring thermal contact resistance of graphite thin film
}

\section{materials}

\author{
Xiaogang $\mathrm{Li}^{1}$, Renxi Luo ${ }^{1}$, Weifang Zhang ${ }^{1}$, Haitao Liao ${ }^{2, *}$ \\ ${ }^{1}$ School of Reliability and Systems Engineering, Beihang University, Beijing, China \\ ${ }^{2}$ Department of Industrial Engineer, University of Arkansas, Fayetteville, AR, US \\ *Corresponding author: E-mail: liao@uark.edu
}

\begin{abstract}
Thermal contact resistance (TCR) is an important parameter in thermal analysis of materials. Because of many influential factors, it is difficult to find a general model or computational formula to calculate the TCR of a solid interface. In many engineering applications, TCR values are usually obtained through experiments. Unlike extensive research focusing on ordinary columnar materials, this paper aims at measuring the TCR values of graphite thin film materials. The technical challenge is that it is not convenient to embed thermocouples into such materials. To overcome this challenge, a steady-state method using a copper heat flux meter is developed, which provides a useful tool for indirect TCR measurement. In our experiments, the TCR values of the graphite thin film materials are successfully measured under different temperature and pressure levels. The results provide a valuable guideline for the use of this type of material in high-temperature, high-pressure applications.
\end{abstract}

Keywords: Thermal contact resistance; Graphite thin film materials; Heat flux; Interface temperature drop; Scanning electron microscope

\section{Introduction}

Thermal contact resistance (TCR) is used to characterize heat transfer across an interface. Its reciprocal is called thermal contact conductance (TCC). It is an important parameter in thermal analyses of structural components used in aerospace, automotive, metalworking, and nuclear industries [1-4]. In recent years, many studies have shown that catastrophic failures of heat transfer components are related to TCR between the component materials and media. 
As a result, how to accurately measure TCR is of great importance in materials science and engineering. In thermal design and analysis of complex structures, the needs are more vital as the uncertainty in TCR measurements may significantly affect the analysis results and mislead a series of development efforts.

In a nuclear power plant, the nuclear reactor is required to transfer heat to the outside environment through thin film materials for cooling. When two structural components are in contact with each other, microscopic imperfect contact cannot be avoided. Such contact surface is actually made of a large number of discrete and small contacting spots separated by relatively large gaps. Because the thermal resistance of the materials at contacting spots is different from that of the media in those gaps, there will be a thermal constriction when the heat transfers across the interface of contact materials [5]. In other words, there will be resistance (i.e., TCR) to heat flux in such a heat transfer process.

Some theoretical models and empirical (or semi-empirical) formulas for TCR calculation have been reported in the literature, such as the elastic model [6], plastic model [7], and the elastoplastic model [8]. Barzelay et al. [9] and Sridhar and Yovanovich [10] provided detailed literature reviews of such theoretical models. However, there are many factors affecting the TCR, so it is difficult to find a general computational model or formula for calculating the TCR of any solid interface. Because the models or formulas are quite different under different contact conditions, when conducting thermal analysis and design the TCR values of a solid interface are usually obtained through a series of experiments.

Milanez et al. [11] studied the TCR for aluminum and stainless-steel contact interface using a piece of self-developed equipment. Rosochowska et al. [12] used a sandwich approach to measure and calculate the TCC between forging work-material and tools using a steady-state method. Yeh and Chu studied the TCR measurement of aluminum honeycombs [13]. Yovanovich tested the TCR of ground/lapped stainless steel interfaces [14]. Wang et al. [15] developed an experimental apparatus to measure the TCC of the interface between two solid materials in the form of round rod with the help of compensation heaters. Pressed pairs of $\mathrm{GH} 4169 / \mathrm{K} 417$ contacts in the range of $160 \sim 280{ }^{\circ} \mathrm{C}$ and $60 \sim 180 \mathrm{MPa}$ were used to demonstrate the applicability of the apparatus. Liu et al. [16] tested the high-temperature TCR between a high thermal conductivity C/C material and superalloy Inconel 600 under 
different interface conditions. Cylindrical specimens $(30 \mathrm{~mm}$ in diameter and $40 \mathrm{~mm}$ in length) were made from Inconel 600 and high thermal conductivity C/C composites material.

In critical applications where high TCC is desired, soft, thin film materials with high conductivity can be used at contacting surfaces to enhance heat transfer. In the literature, extensive research has been conducted on columnar materials, but quite limited studies have been reported on TCR measurement of thin film materials. One of the big challenges is that it is not convenient to embed temperature sensors in such a thin film material. This paper reports a viable method for indirectly measuring the TCR of thin film materials. In particular, equipment and a steady-state method are developed to measure the TCR of graphite thin film materials under different temperature and pressure levels. It is expected that the indirect TCR measurement tool will be widely used in other applications involving thin film materials.

The remainder of this paper is organized as follows. Section 2 introduces the steady-state method and the rationale behind the technique for the TCR measurement of thin film materials. Experimental study of TCR of graphite thin film materials is provided in Section 3. Section 4 analyzes the experimental results and discusses several practical aspects of this work. Section 5 concludes this paper.

\section{Thermal contact resistance measurement of thin film materials}

\subsection{Steady-state method}

The TCR of a material is defined as

$$
R=\Delta T / q
$$

where $R$ is the TCR measured in $\mathrm{m}^{2} \cdot \mathrm{K} / \mathrm{W}, \Delta T$ is the interface temperature drop measured in $\mathrm{K}$, and $q$ is the heat flux density measured in $\mathrm{W} / \mathrm{m}^{2}$. The steady-state method has been widely used in TCR measurement by disposing temperature sensors in test specimens. However, it is extremely difficult, if not impossible, to realize that for thin film materials. To overcome this challenge, a technique for measuring the TCR of thin film materials based on the steady-state method and a copper heat flux meter is proposed.

Technically, this method calculates the TCR by measuring the temperature changes 
caused by heat transfer within the interface of different materials. Because the temperature sensor and the surrounding medium need sufficient heat exchange, it will take some time to reach thermal equilibrium. Therefore, the calculation should be performed using the testing data after the experiment has reached the steady state.

\subsection{Heat flux}

When the heat transfers along a certain direction, there is a temperature gradient in the sample along this direction due to the thermal resistance. According to the Fourier's law of heat conduction, the following equation holds

$$
q=-k \mathrm{~d} T / \mathrm{d} x,
$$

where $k$ is the thermal conductivity which generally varies with temperature. In a narrow temperature range, the thermal conductivity satisfies

$$
k(T)=k_{0}(1+T / m),
$$

where $m$ is the related parameter of the slope of thermal conductivity in the temperature changing range, and $k_{0}$ is the ordinate intercept of the extension line of line segment in the temperature changing range. Then, $k_{0} / m$ is the slope of thermal conductivity in the temperature changing range.

If the thermal conductivity of a heat-flow meter and the temperature gradient are known, the axial heat flux within the heat-flow meter and test samples can be calculated. The Fourier's law with changing thermal conductivity coefficient can be obtained from Eqs. (2) and (3), and the integral of this Fourier's law expression results in the axial heat flux expression. If the lateral heat loss of samples is ignored, the axial heat flux density is

$$
q=\left(T_{1}-T_{n}\right) k_{0}\left[1+\left(T_{1}+T_{n}\right) / 2 m\right] / s,
$$

where $T_{1}$ is the temperature at the first test point in the heat flow meter sample, $T_{n}$ is the temperature at the $n$th test point, and $s$ is the distance between the first test point and the $n$th test point. In the experiment, it is needed to first calculate the values of $k_{0}$ and $m$ in Eq. (3) for thermal conductivity under varying temperature, and then to calculate the value of heat-flow according to the temperature and the distance between the test points. 


\subsection{Interface temperature drop}

This experiment requires a heat flux meter, two columnar specimens and a thin film sample. They are stacked in the following order (see Fig. 1): one columnar specimen $\rightarrow$ thin film specimen $\rightarrow$ another columnar specimen $\rightarrow$ the heat flow meter, and are vertically and axially arranged between the bottom of heating device and the top of stress load device. Moreover, the copper heat flux meter and the two columnar specimens are equipped with thermocouples. The thermocouples are connected with a data acquisition system used to collect the axial temperatures of the specimens. Fig. 1 illustrates the interface temperature drop of testing samples and the possibility for performing extrapolation. Note that $T_{C l}$ and $T_{C 2}$ are the interface temperatures of the columnar sample 1 and columnar sample 2 contacting with the thin films material, respectively.

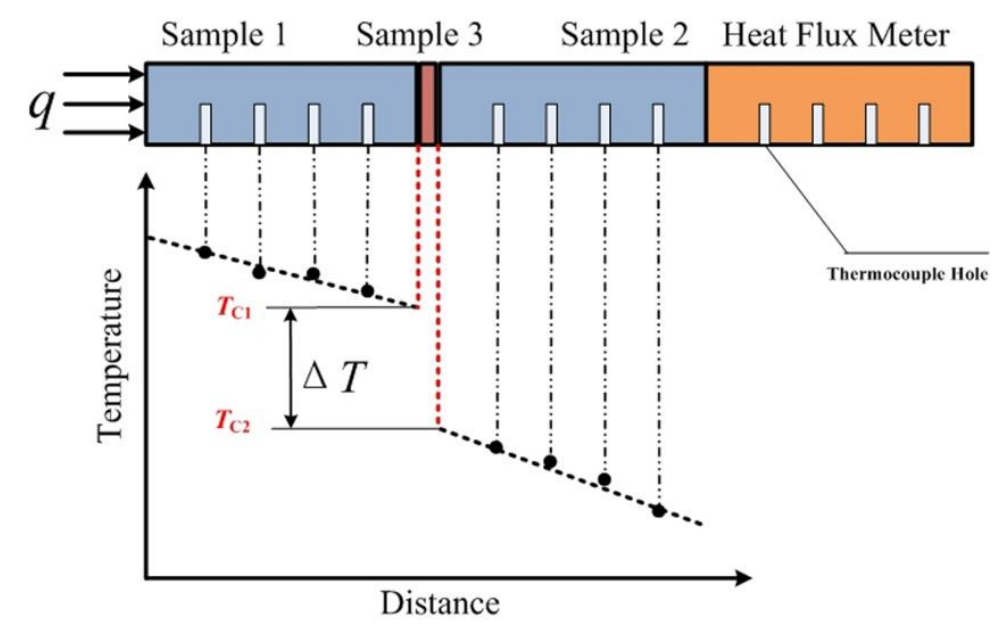

Fig. 1. Illustration of interface temperature drop.

In this experiment, the heat insulation system and the compensated heating system are used to maximize the realization of the axial one-dimensional heat conduction of the specimens, so the heat transfer in the experiment can be treated as a one-dimensional steady-state problem. As the change of material thermal conductivity is little in the test, in the allowed range of error the temperature distribution can be approximately considered having a linear distribution.

From Eq. (4), the temperature of interest $T$ can be obtained by

$$
T=\sqrt{\left(m+T_{i}\right)^{2}-2 q m x_{i} / k_{0}}-m,
$$


where $T_{i}$ is the measured temperature at the $i$ th point, and $x_{i}$ is the distance from the $i$ th test point to the interface. To this end, the thermal conductivity of the columnar specimens under different temperatures is tested, and then the values of $k_{0}$ and $m$ in Eq. (4) are calculated. Afterwards, the interface temperature drop of the columnar specimens is obtained by solving a least-square optimization problem.

Technically, for the interface temperature $T_{C 1}$ and $T_{C 2}$ of the columnar specimens, the following optimization problem can be formulated

$$
\begin{aligned}
& \min \quad f(T)=\sum_{i=1}^{n}\left[\sqrt{(m+T)^{2}-2 q m x_{i} / k_{0}}-m-T_{i}\right]^{2} \\
& \text { s.t. } \quad T_{\mathrm{a}} \leq T \leq T_{b}
\end{aligned}
$$

where $T$ is the temperature of interface desired, $T_{i}$ is the measured temperature at the $i$ th point, $x_{i}$ is the distance from the $i$ th test point to the interface, and $T_{a}$ and $T_{b}$ specify the temperature range according to the temperature test results.

From Eq. (6), the interface temperature $T_{C 1}$ and $T_{C 2}$ of columnar specimens can be calculated, and then the interface temperature drop $\Delta T$ is obtained.

\subsection{TCR between columnar and thin film specimen}

The thermal resistance of thin film sample can be calculated according to the thermal conductivity $k_{B}$ at the corresponding temperature and the thickness $\delta$ of the thin film sample. Let $r$ be the thermal resistance of the thin film defined by

$$
r=\delta / k_{B}
$$

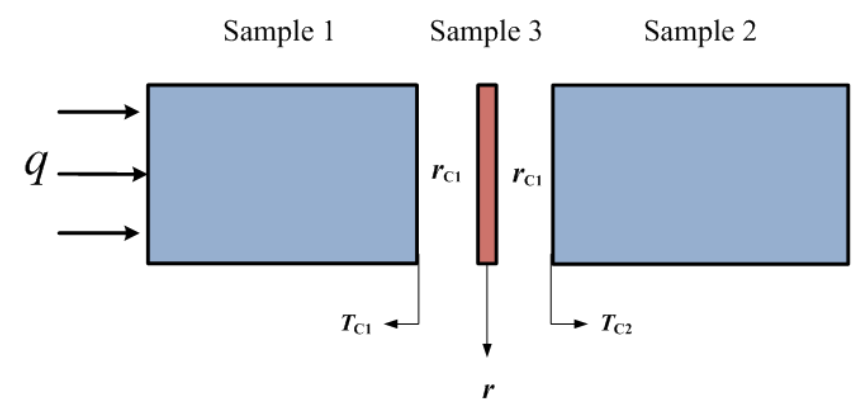

Fig. 2. Set-up with two columnar specimens and a thin film. 
Fig. 2 shows how the TCR of columnar and thin film specimen is measured. For the two contact interfaces of columnar specimens and thin film specimen, the surface roughness values in the two sides of thin film specimen are approximately equal. Moreover, because the difference between $T_{C 1}$ and $T_{C 2}$ is small, the influence of the direction of heat flow on TCR can be ignored, and thus the TCR values of the two contact interfaces between the two columnar specimens and the thin film specimen are considered equal. As a result, we have

$$
\left(T_{C 1}-T_{C 2}\right) / q=2 r_{C 1}+r
$$

where $r_{C 1}$ is the TCR between the columnar specimen and thin film specimen (considering the same for both sides), which can be calculated by

$$
r_{C 1}=\frac{1}{2}\left(\frac{T_{C 1}-T_{C 2}}{q}-\frac{\delta}{k_{B}}\right)
$$

\subsection{TCR between thin film specimens}

To measure the TCR between two thin film specimens, $i$ pieces of thin film specimens were placed between the two columnar specimens (see Fig. 3).

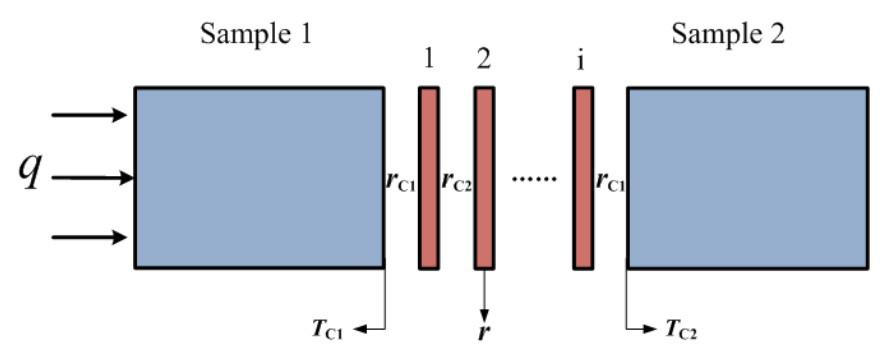

Fig. 3. Set-up with two columnar specimens and multiple thin films.

Again, by ignoring the influence of the direction of heat flow on the values of TCR in the stack of materials, we have

$$
\left(T_{C 1}-T_{C 2}\right) / q=2 r_{C 1}+i r+(i-1) r_{C 2},
$$

where $i$ is the number of thin film specimens between the two columnar samples 1 and 2 , $T_{C 1}$ is the surface temperature of columnar sample $1, T_{C 2}$ is the surface temperature of columnar sample 2, $r$ is the thermal resistance of thin film specimen itself, $r_{C 1}$ is the TCR of 
columnar specimen and thin film specimen which can be calculated from Eq. (9), and $r_{C 2}$ is the TCR of adjacent thin film specimens. Using Eq. (10) and the TCR between the thin film and the columnar material obtained from Eq. (9), the TCR between the thin film specimens can be calculated.

\section{Experimental study of TCR of graphite thin film materials}

\subsection{Experimental setup}

A copper heat flux meter, graphite columnar specimens and graphite thin film samples were used to demonstrate this method for measuring the TCR between the graphite thin film materials. Fig. 4 shows the testing system.

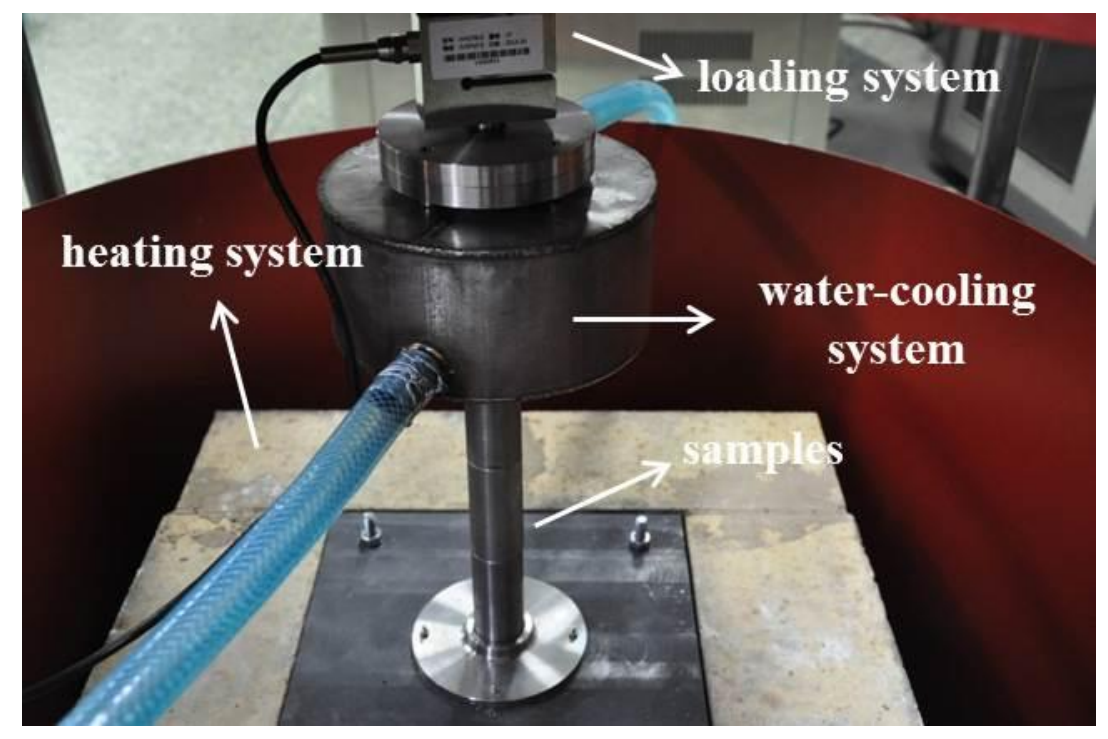

Fig. 4. Experimental set-up

The equipment consists of a heating system, heat insulation system, compensated heating system, water-cooling system, loading system, and data acquisition system. The heating system is composed of a hollow stove and a heating block, which is located on the substrate of the device. The heat insulation system containing ceramic fiber is designed to ensure one-dimensional heat conduction in the experiment, and to reduce the experimental error. The compensated heating system consists of three circular heaters. Through the measurements from the temperature sensors, the temperature of the heater is set to be the average temperature of the specimen interface for the test. In the radial direction of the 
contact interface, the heat transfer reaches the equilibrium state, and it can avoid the loss of lateral heat flux. The water-cooling system is made up of a circulating water tank and inlet and outlet pipes, and is installed on the top of the test samples to increase the temperature drop at the two ends of the samples. The water-cooling system can speed up the process of heat transfer, and increase the longitudinal heat flow to reduce the deviation caused by lateral heat loss. The loading system is achieved by tightening the nut on the top of the roof. A pressure sensor is fixed at the center of the roof. By tightening the nut on the stud bolt through the top of the roof, the downward pressure will be loaded on the roof, which is transferred to the samples. The pressure sensor installed under the roof is to measure the pressure. The data acquisition system includes thermocouples, inspection instrument and a computer.

In the thermal contact resistance testing experiment, temperature acquisition from the thermocouples, the distance between the measuring points and lateral heat loss will cause an experimental error. To quantify the error, our previous work [15] shows that the errors of thermal conductivity calibration, temperature inspection instrument and thermocouple temperature measurement are $1.8 \%, 0.2 \%$ and $0.8 \%$, respectively. Moreover, according to the preliminary test and calculation, the error caused by the heat loss of equipment is $2 \%$. Considering the law of error propagation, the test error caused by the heat transfer is about 4.8\%. In addition, the error in the process of temperature drop measurement is $7.8 \%$. The total error of TCR is $9.2 \%$, which is controlled within $10 \%$.

\subsection{Experimental specimens}

The thin film material studied in this paper is graphite thin film. The material of columnar specimens belongs to a special graphite. The copper heat flow meter and special graphite cylindrical materials were processed into a cylindrical form with size $\phi 30 \times 65 \mathrm{~mm}$. Four radial thermocouple mounting holes were processed into each sample, and the size of each hole is $\phi 1.5 \times 15 \mathrm{~mm}$. The distance between the adjacent holes is $15 \mathrm{~mm}$. Fig. 5 shows the details of the columnar specimen. Graphite thin films (see Fig. 6) are processed into $\phi 30$ $\times 0.38 \mathrm{~mm}$ circular sheet. The roughness of the graphite foil surfaces that contact the two graphite columnar specimens were made consistent as much as possible. 


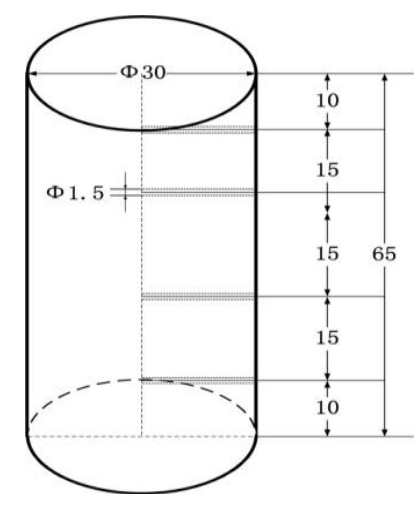

Fig. 5. Columnar specimen with four radial thermocouple mounting holes.

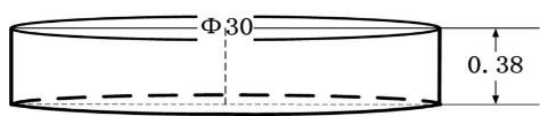

Fig. 6. Graphite thin film sample.

\section{Results and discussion}

In the first step, the TCR values between the graphite columnar specimens and graphite thin film sample for $373.15 \mathrm{~K}$ and pressures of $1 \mathrm{MPa}, 2.5 \mathrm{MPa}, 5 \mathrm{MPa}, 7.5 \mathrm{MPa}$ and $10 \mathrm{MPa}$, respectively, were measured, and then the experiments were repeated for $473.15 \mathrm{~K}$ and $573.15 \mathrm{~K}$.

The temperature of the sample contact surface cannot be directly measured, so we did not measure the temperature of a particular point on the surface. In order to ensure that the interface temperature is the predetermined value of $373.15 \mathrm{~K}, 473.15 \mathrm{~K}$ and $573.15 \mathrm{~K}$ up and down in a smaller range, the average value of the temperature of the two thermocouples adjacent to the contact interface the will be set to the value of the test.

Parameters $k_{0}=398$ and $m=-8468$ in Eq. (3) of the copper heat flux meter were calculated by thermal conductivity for varying temperature as well as the value of heat-flow density according to the temperature of test points and the distance of test points. Solving the problem given in Eq. (6), the values of the interfaces temperatures $T_{C 1}$ and $T_{C 2}$ of columnar specimens were calculated, and then the interface temperature drop of the two graphite columnar specimens $\Delta T=T_{C 1}-T_{C 2}$ was obtained. 
The thermal resistance of the graphite thin film sample used in the experiment is equal to $3.8 \mathrm{E}-6 \mathrm{~K} / \mathrm{W}$. Using all these values obtained from the preceding calculations and Eq. (8), the TCR values between the graphite columnar specimens and graphite thin film sample were obtained. To reduce the impact of experimental errors, three replications were conducted for each condition, and the average results are presented in Table I.

TABLE I. TCR values $\left(\mathrm{m}^{2} \cdot \mathrm{K} / \mathrm{W}\right)$ between columnar specimens and a graphite thin film.

\begin{tabular}{|c|c|c|c|}
\hline & $373.15 \mathrm{~K}$ & $473.15 \mathrm{~K}$ & $573.15 \mathrm{~K}$ \\
\hline $1 \mathrm{MPa}$ & $6.4244 \mathrm{E}-05$ & $4.8484 \mathrm{E}-05$ & $4.1276 \mathrm{E}-05$ \\
\hline $2.5 \mathrm{MPa}$ & $3.8612 \mathrm{E}-05$ & $4.4641 \mathrm{E}-05$ & $3.8414 \mathrm{E}-05$ \\
\hline $5 \mathrm{MPa}$ & $3.1028 \mathrm{E}-05$ & $4.0554 \mathrm{E}-05$ & $3.3314 \mathrm{E}-05$ \\
\hline $7.5 \mathrm{MPa}$ & $2.9658 \mathrm{E}-05$ & $3.7376 \mathrm{E}-05$ & $3.3518 \mathrm{E}-05$ \\
\hline $10 \mathrm{MPa}$ & $2.8114 \mathrm{E}-05$ & $3.2838 \mathrm{E}-05$ & $3.2512 \mathrm{E}-05$ \\
\hline
\end{tabular}

At the second step, five pieces of graphite thin film specimens were placed between the two columnar specimens. The TCR values between graphite thin film specimens for $373.15 \mathrm{~K}$ and pressures of $1 \mathrm{MPa}, 2.5 \mathrm{MPa}, 5 \mathrm{MPa}, 7.5 \mathrm{MPa}$ and $10 \mathrm{MPa}$, respectively, were first measured, and then the experiments were repeated for $473.15 \mathrm{~K}$ and $573.15 \mathrm{~K}$.

Similar to the first step, the heat-flow density values and the values of interface temperature drop of the graphite thin film specimens were calculated. At last, the TCR values between graphite thin film specimens were obtained. Table II shows the average TCR values for three replications each.

TABLE II. TCR values $\left(m^{2} \cdot \mathrm{K} / \mathrm{W}\right)$ between graphite thin films.

\begin{tabular}{|c|c|c|c|}
\hline & $373.15 \mathrm{~K}$ & $473.15 \mathrm{~K}$ & $573.15 \mathrm{~K}$ \\
\hline $1 \mathrm{MPa}$ & $6.5216 \mathrm{E}-05$ & $5.2607 \mathrm{E}-05$ & $5.1515 \mathrm{E}-05$ \\
\hline $2.5 \mathrm{MPa}$ & $6.1814 \mathrm{E}-05$ & $5.0774 \mathrm{E}-05$ & $4.5258 \mathrm{E}-05$ \\
\hline $5 \mathrm{MPa}$ & $5.9526 \mathrm{E}-05$ & $4.6702 \mathrm{E}-05$ & $4.0430 \mathrm{E}-05$ \\
\hline $7.5 \mathrm{MPa}$ & $5.2711 \mathrm{E}-05$ & $4.3450 \mathrm{E}-05$ & $3.6001 \mathrm{E}-05$ \\
\hline $10 \mathrm{MPa}$ & $5.1088 \mathrm{E}-05$ & $4.3054 \mathrm{E}-05$ & $3.3617 \mathrm{E}-05$ \\
\hline
\end{tabular}

As shown in Table II, at each temperature level, the TCR value of graphite thin film decreases gradually as the pressure increases. On the other hand, under a specific pressure 
level, the TCR value decreases as the temperature increases. The curves in Fig. 7 illustrate the trend of TCR under different levels of temperature and pressure. One can see that the TCR decreases rapidly as the interfacial stress increases. When the load goes beyond 7.5MPa, the change of TCR reduces.

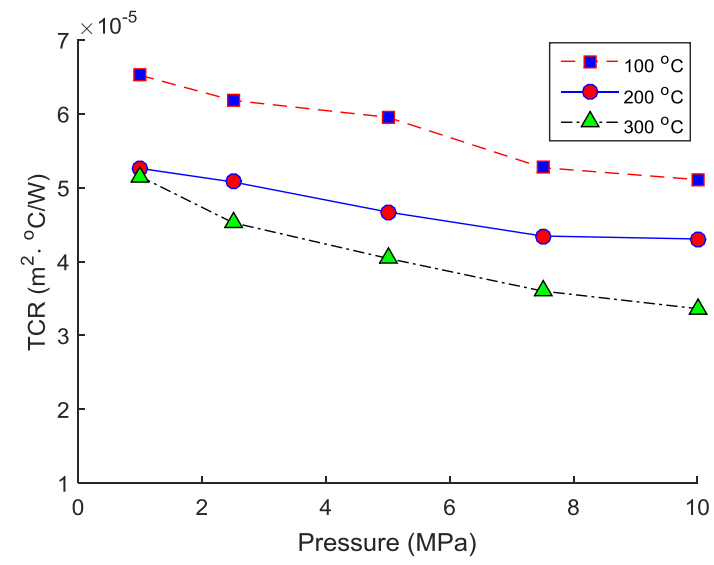

Fig. 7. TRC between graphite thin films under different levels of temperature and pressure.

The graphite thin films before and after the experiment conducted at $473.15 \mathrm{~K}$ were inspected. The pictures obtained using a scanning electron microscope $(50 \times$ and $200 \times$ magnifications) are shown in Fig. 8. The pictures on the left are the graphite samples before the experiment, and the ones on the right are the samples after the experiment. The two pictures at the bottom show the different distributions of local peaks of the material $(200 \times$ magnification). Clearly, the graphite thin films after the experiment became much smoother. 

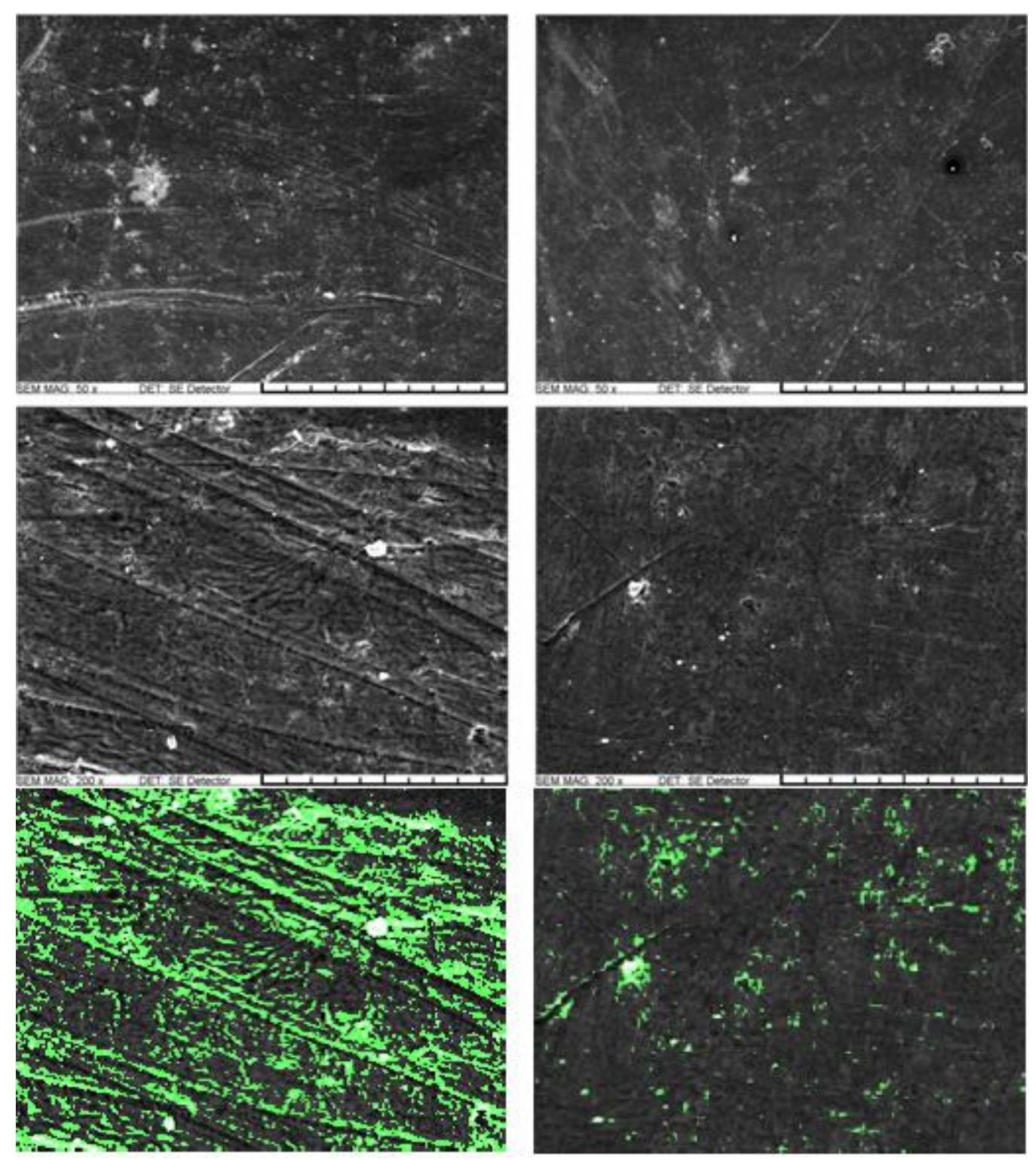

(a) before the experiment

(b) after the experiment

Fig. 8. Magnified views of graphite thin film under $50 \times$ and $200 \times$ magnifications.

Based this observation, the results shown in Fig. 7 can be interpreted as follows. At the initial stage of loading, although the load is relatively small, because the surface of the new material is still rough, each roughness peak on average will bear more pressure. This will result in plastic and elastic deformation making the actual contacting area rapidly increase. As the interface load increases, the number of deformation of interface micro bulge will increase. As a result, the actual contacting area increases leading to the decrease in TCR. When the pressure is increased to a certain level (above $7.5 \mathrm{MPa}$ ), the number of deformed interface micro bulges gradually becomes saturated, and the interface deformation degree is becoming 
smaller and smaller. As a result, the increment of contacting area slows down making the TCR decrease more and more slowly.

\section{Conclusion}

Based on the steady-state method for TCR measurement, this paper presents an indirect method to measure the TCR of thin film materials. This method resolves the difficulty of embedding thermocouples into such materials. Technically, the method consists of two steps. The TCR between the thin film material and the columnar material is tested followed by the measurement of the TCR between the thin film materials. The method is applied to measuring the TCR between graphite film materials, which has not been reported in the literature. In particular, the influences of load pressure and temperature on the TCR are analyzed. Because such thin film materials have been utilized in many hot components, this work provides a useful measurement tool in the area of thermal analysis and benefits the related industry where the safety and reliability of structural materials are vitally important.

\section{Acknowledgements}

Dr. Liao's work was partially supported by the U.S. National Science Foundation under grant \#CMMI 1238301. The authors would also like to thank the reviewers and editors for providing valuable comments and suggestions that improved the presentation of this paper.

\section{References}

[1] Grujicic, M., Zhao, C.L. and Dusel, E.C., The effect of thermal contact resistance on heat management in the electronic packaging, Appl. Surf. Sci., 246 (1-3) (2005) 290-302.

[2] Fletcher, L.S., Recent developments in contact conductance heat transfer, J. Heat Transfer, 110 (1988) 1059-1070.

[3] Lambert, M.A. and Fletcher, L.S., Thermal contact conductance of spherical rough metals, J. Heat Transfer, 119 (1997) 684-690. 
[4] Fieberg, C. and Kneer, R., Determination of thermal contact resistance from transient temperature measurements, International Journal of Heat and Mass Transfer, 51(5-6) (2008) 1017-1023.

[5] Wang, Z., Zhang, W., Tang, Q., and Hou, W, Progress in thermal contact resistance, Rare Metal Materials and Engineering, 40(S3) (2011) 211-215.

[6] Mikic, B.B., Thermal contact conductance: theoretical considerations, Int. J. Heat Mass Transfer, 17(2) (1974) 205-214.

[7] Cooper, M.G., Mikic, B.B. and Yovanovich, M.M., Thermal contact conductance, Int. J. Heat Mass Transfer, 12(3) (1969) 279-300.

[8] Sridhar, M. and Yovanovich, M., Elastoplastic contact conductance model for isotropic conforming rough surfaces and comparison with experiments, J. Heat Transfer, 118(1) (1996) 3-9.

[9] Barzelay, M.E., Tong, K.N. and Holloway, G.F., Effect of pressure on thermal conductance of contact joints, Report No. NACA-TN-3295, Property Fairchild Engineering Library, Washington, May 20, 1955, pp. 1-52.

[10] Sridhar, M. and Yovanovich, M., Critical review of elastic and plastic thermal contact conductance models and comparison with experiment, The 28th AIAA Thermophysics Conference, Orlando, FL, July 6-9, 1993.

[11] Milanez, F., Culham, J. R. and Yovanovich, M. M., Experimental study on the hysteresis effect of thermal contact conductance at light loads, The 40th AIAA Aerospace Sciences Meeting \& Exhibit, Reno, NV, January 14-17, 2002, pp. 1-8.

[12] Rosochowska, M., Chodnikiewicz, K. and Balendra, R., A new method of measuring thermal contact conductance, J. Mater. Proc. Technol., 145(2) (2004) 207-214.

[13] Yeh, L. T., Chu, R. C., Thermal interface resistance, ASME Press, 2002.

[14] Yovanovich, M. and Nho, K., Experimental investigation of heat flow rate and direction on contact resistance of ground/lapped stainless steel interfaces, The 24th AIAA Thermophysics Conference, Buffalo, NY, June 12-14, 1989.

[15] Wang, Z.R., Yang, J. and Wang, S., Compensation heating technique for experimental investigation of thermal contact conductance across GH4169/K417 interface, Rare Metal Materials and Engineering, 42(8) (2013) 1572-1575. 
[16] Liu, D., Luo, Y. and Shang, X., Experimental investigation of high temperature thermal contact resistance between high thermal conductivity C/C material and Inconel 600, International Journal of Heat and Mass Transfer, 80 (2015) 407-410.

\section{Nomenclature}

$R$ - thermal contact resistance (TCR)

$\Delta T$ the interface temperature drop

$q$ the heat flux density

$k-$ the thermal conductivity which generally varies with temperature

$m$ the related parameter of the slope of thermal conductivity in the temperature changing range

$k_{0}$ the ordinate intercept of the extension line of line segment in the temperature changing range

$T_{1}$ the temperature at the first test point in the heat flow meter sample

$T_{n} \longrightarrow$ the temperature at the $n$th test point

$s —$ the distance between the first test point and the $n$th test point

$T_{C l} \longrightarrow$ the interfaces temperature of the columnar sample 1 contacting with thin films material

$T_{C 2} \longrightarrow$ the interfaces temperature of the columnar sample 2 contacting with thin films material

$T_{i} \longrightarrow$ the measured temperature at the $i$ th point

$x_{i} \longrightarrow$ the distance from the $i$ th test point to the interface

$T_{a}, T_{b} \longrightarrow$ the temperature range according to the temperature test results

$r-$ be the thermal resistance of the thin film

$k_{B}$ the thermal conductivity at the corresponding temperature of the thin film sample

$\delta \_$the thickness of the thin film sample

$r_{C 1} \longrightarrow$ the TCR between the columnar specimen and thin film specimen (considering the same for both sides)

$r_{C 2} \longrightarrow$ the TCR of adjacent thin film specimens 